Eduardo Carvalho Pessoa ${ }^{1}$

José RICARDo Paciência RodrIGUes ${ }^{2}$

Carla Prisclla Kamita Carvalho Pessoa ${ }^{3}$

Heloísa Maria de luca Véspoli ${ }^{4}$

Gilberto Uemura ${ }^{2}$

\title{
Punção aspirativa de linfonodo axilar guiada pela ultrassonografia é eficaz como método de predição de acometimento linfonodal em pacientes com câncer de mama?
}

\author{
Axillary ymph node aspiration guided by ultrasound is effective as a method \\ of predicting lymph node involvement in patients with breast cancer?
}

Artigo Original

\begin{abstract}
Palavras-chave
Biopsia por agulha fina Neoplasias da mama/ultrassonografia Linfonodos/citologia

Linfonodos/diagnóstico Linfonodos/ultrassonografia Biopsia de linfonodo sentinela

Keywords

Biopsy, fine needle Breast neoplasms/ultrasonography Lymph nodes/cytology Lymph nodes/diagnosis Lymph nodes/ultrasonography

Sentinel lymph node biopsy
\end{abstract}

\section{Resumo}

OBJETIVO: Avaliar a viabilidade e acurácia diagnóstica da ultrassonografia pré-operatória combinada com biopsia por agulha fina (US-PAAF) e do exame clínico da axila em pacientes com câncer de mama. MÉTODOS: Neste estudo prospectivo 171 axilas de pacientes com câncer de mama foram avaliadas pelo exame clínico e ultrassonografia (US) com e sem biopsia por agulha fina (PAAF). Os linfonodos com espessura cortical maior que 2,3 mm na ultrassonografia foram considerados suspeitos e submetidos a US-PAAF. RESULTADOS: A análise de regressão logística não mostrou correlação estatisticamente significativa entre exame clínico e axilas positivas no exame patológico. Em relação à avaliação axilar com US, o risco de achados anatomopatológicos positivos aumentou 12,6 vezes, valor Kappa de Cohen foi de 0,12 para exame clínico, 0,48 para US e 0,80 para US-PAAF. A acurácia foi de 61,4\% para o exame clínico, 73, 1\% para os US e 90, 1\% para US-PAAF. Análise Receiver Operating Chracteristics (ROC) mostrou que uma espessura de 2,75 mm cortical correspondeu à mais elevada sensibilidade e especificidade na predição metástase axilar (82,7 e 82,2\%, respectivamente). CONCLUSÕES: A US combinada com aspiração por agulha fina é mais precisa que o exame clínico na avaliação do status axilar no pré-operatório em mulheres com câncer de mama. Aquelas que são US-PAAF positivo podem ser direcionadas para esvaziamento linfonodal axilar imediatamente, e somente aqueles que são US-PAAF negativos devem ser considerados para biópsia de linfonodo sentinela.

Abstract

PURPOSE: To assess the feasibility and diagnostic accuracy of preoperative ultrasound combined with ultrasound-guided fine-needle aspiration (US-FNA) cytology and clinical examination of axillary lymph node in patients with breast cancer. METHODS: In this prospective study, 171 axillae of patients with breast cancer were evaluated by clinical examination and ultrasonography (US) with and without fine needle aspiration (FNA). Lymph nodes with maximum ultrasonographic cortical thickness $>2.3 \mathrm{~mm}$ were considered suspicious and submitted to US-FNA. RESULTS: Logistic regression analysis showed no statistically significant correlation between clinical examination and pathologically positive axillae. However, in axillae considered suspicious by ultrasonography, the risk of positive anatomopathological findings increased 12.6-fold. Cohen's Kappa value was 0.12 for clinical examination, 0.48 for US, and 0.80 for US-FNA. Accuracy was $61.4 \%$ for clinical examination, 73. 1\% for US and 90. 1\% for US-FA. Receiver Operating Characteristics (ROC) analysis demonstrated that a cortical thickness of $2.75 \mathrm{~mm}$ corresponded to the highest sensitivity and specificity in predicting axillary metastasis (82.7 and 82.2\%, respectively). CONCLUSIONS: Ultrasonography combined with fine-needle aspiration is more accurate than clinical examination in assessing preoperative axillary status in women with breast cancer. Those who are US-FNA positive can be directed towards axillary lymph node dissection straight away, and only those who are US-FNA negative should be considered for sentinel lymph node biopsy.
Correspondêncio

Eduardo Carvalho Pesso Distrito de Rubião Junior, $s / n$ CEP: 18618970

Botucatu (SP), Brasil

Recebido

$19 / 12 / 2013$

Aceito com modificaçōes

$27 / 01 / 2014$
Centro de Avaliação em Mastologia "Professor Laurival Antonio De Luca" da Faculdade de Medicina de Botucatu da Universidade Estadual Paulista "Júlio de Mesquita Filho" - UNESP - Botucatu (SP), Brasil.

'Centro de Avaliação em Mastologia, Setor de Diagnóstico por Imagem da Disciplina de Mastologia, Faculdade de Medicina de Botucatu, Universidade Estadual Paulista "Júlio de Mesquita Filho" - UNESP - Botucatu (SP), Brasil.

${ }^{2}$ Setor de Mastologia, Departamento de Ginecologia Obstetrícia e Mastologia, Faculdade de Medicina de Botucatu, Universidade Estadual Paulista "Júlio de Mesquita Filho" - UNESP - Botucatu (SP), Brasil.

${ }^{3}$ Centro de Ciências da Saúde, Universidade de Caxias do Sul - UUCS - Caxias do Sul (RS), Brasil.

${ }^{4}$ Departamento de Ginecologia Obstetrícia e Mastologia, Faculdade de Medicina de Botucatu, Universidade Estadual Paulista "Júlio de Mesquita Filho" - UNESP - Botucatu (SP), Brasil.

Conflito de interesses: não há. 


\section{Introdução}

Recentemente foi introduzida a biópsia do linfonodo sentinela (BLS) como novo método de avaliação axilar. O linfonodo sentinela é definido como o primeiro linfonodo a receber a drenagem linfática de um câncer de mama, e por essa razão será o primeiro sítio a receber metástases se ocorrer disseminação por essa via ${ }^{1}$. A BLS é considerada método de amostragem axilar seletiva, minimamente invasivo e altamente sensível para a identificação de metástases. O principal critério para selecionar as pacientes que serão submetidas ao linfonodo sentinela é a avaliação clínica da axila. No entanto, o exame clínico é pouco sensível para avaliar o acometimento linfonodal axilar, pois é observado que de 15 a $60 \%$ das pacientes com axila clinicamente negativa têm linfonodos positivos, em média de 30 a $40 \%$ de linfonodos sentinelas metastáticos ${ }^{1-7}$.

A ultrassonografia (US) pode detectar linfonodos suspeitos em axilas clinicamente negativas e selecionar as pacientes para a biopsia por agulha, reduzindo o número de biópsias desnecessárias do linfonodo sentinela. Também pode diminuir a taxa de falso-negativos por detectar linfonodos com extenso envolvimento metastático e cujas vias de drenagens estejam bloqueadas, ou seja, que não seriam marcados pelo corante e/ou pelo radiofármaco. Trabalhos mostram uma redução em $15 \%$ do risco de falso negativo do linfonodo sentinela quando se associa o uso da ultrassonografia ao da biopsia por agulha fina (US-PAAF) ${ }^{8,9}$.

Os primeiros estudos utilizando a ultrassonografia como ferramenta para avaliar o estado axilar valorizavam o tamanho dos linfonodos, eram realizados com transdutores de 5 a $7,5 \mathrm{MHz}$, limitando a visualização de detalhes da anatomia linfonodal. Eram considerados linfonodos suspeitos pela ultrassonografia aqueles com tamanho maior que $5 \mathrm{~mm}^{10-12}$. Atualmente sabemos que o tamanho não deve ser critério para definir presença de neoplasia ou não do linfonodo, pois encontramos linfonodos com $5 \mathrm{~cm}$ livres de metástases e linfonodos de $5 \mathrm{~mm}$ comprometidos ${ }^{13}$.

Com o avanço técnico dos aparelhos e a utilização de transdutores lineares com frequência até $12 \mathrm{MHz}$, a partir de 1996 foram publicados trabalhos em que critérios morfológicos e estruturais dos linfonodos começaram a ser avaliados como parâmetros de malignidade ou benignidade. Os primeiros critérios descritos foram a forma e a ecogenicidade. Os linfonodos com forma redonda e hipoecogênicos eram considerados suspeitos de acometimento metastático ${ }^{14-16}$.

Porém quando estudamos a anatomia do linfonodo entendemos que esses critérios são de aparecimento tardio. Levando em consideração a fisiologia da metástase linfonodal, as células se alojam primeiro na cortical, dilatando-a e distendendo-a. Assim, independente do tamanho ou da forma do linfonodo, observamos as alterações e irregularidades na sua região cortical. Dessa forma, os estudos a partir de 1998 valorizavam as alterações na cortical do linfonodo como critério para sua classificação como suspeito ou não para metástases ${ }^{17}$.

Este trabalho pretende avaliar a acurácia da ultrassonografia pré-operatória combinada com a biópsia por agulha fina para a identificação de metástases nos linfonodos axilares em pacientes com câncer de mama e também avaliar o impacto na redução do número de biópsias do linfonodo sentinela.

\section{Métodos}

Este foi um estudo clínico, prospectivo e analítico, conduzido no período de 2006 a 2008, no qual foram incluídas todas as pacientes com diagnóstico de câncer de mama livres de qualquer tratamento anterior (cirúrgico, radioterápico, quimioterápico e hormonioterápico) e sem apresentar metástases a distância. Foram excluídas pacientes que por algum motivo não puderam ser tratadas cirurgicamente na mama e/ou axila. No total, foram incluídas 168 pacientes para o estudo e avaliadas 171 axilas (3 pacientes apresentarem carcinomas bilaterais).

A média de idade das pacientes foi de 56,8 anos, com variação entre 28 e 88 anos. Os tipos histológicos mais frequentes foram o carcinoma ductal, que correspondeu a $86 \%$, e o carcinoma lobular, com 5,2\% das lesões. Quanto ao tamanho tumoral, observamos que $54,5 \%$ das lesões apresentavam até $2 \mathrm{~cm}, 35,6 \%$ entre 2 e $5 \mathrm{~cm}$ e 9,9\% tinham diâmetro maior que $5 \mathrm{~cm}$.

O projeto foi aprovado pelo Comitê de Ética em Pesquisa da Faculdade de Medicina de Botucatu da Universidade Estadual Paulista "Júlio de Mesquita Filho" - UNESP. Todas as pacientes deste estudo assinaram o termo de consentimento livre e esclarecido.

Inicialmente as pacientes foram submetidas à avaliação clínica da axila por meio da palpação axilar pelo mastologista. Foram classificadas como axilas positivas aquelas que apresentavam linfonodos axilares palpáveis e endurecidos e como axilas negativas aquelas que não apresentavam linfonodos palpáveis.

As pacientes também foram submetidas à avaliação ultrassonográfica da axila, realizada com aparelho GE Logic $5^{\circledR}$ e transdutor multifrequencial linear de 7 a $12 \mathrm{MHz}$ com utilização da harmônica. Um médico com experiência em ultrassonografia mamária e axilar realizou todos os exames. Todas as pacientes foram examinadas inicialmente em decúbito dorsal e decúbito lateral $\left( \pm 60^{\circ}\right)$, com o braço posicionado sob a cabeça.

A avaliação ultrassonográfica foi dividida em duas etapas. Na primeira etapa, foi estudada exclusivamente a topografia rotineira do linfonodo sentinela (nível 1 de Berg, inferior ao músculo peitoral menor), procurando identificar 
linfonodos nessa posição. Em relação ao tempo de avaliação axilar, a mediana foi de 6 min (mínimo de 3 e máximo de $15 \mathrm{~min}$ ). A segunda etapa do exame procurou estudar os outros dois níveis de Berg: o nível 2, sob o músculo peitoral menor, e o nível 3, acima desse músculo, procurando identificar os linfonodos desses níveis. Uma vez identificados, os linfonodos foram estudados com dois cortes, um longitudinal no seu maior diâmetro e outro perpendicular a este. Foram visualizados pelo menos dois linfonodos, com mediana de três linfonodos visualizados no total.

A classificação dos linfonodos como positivos ou negativos foi determinada pelo tamanho da sua cortical. Assim, linfonodos que apresentavam a cortical com diâmetro máximo menor ou igual a $2,3 \mathrm{~mm}$ foram considerados negativos. Os linfonodos com diâmetro máximo maior que 2,3 mm da cortical foram considerados suspeitos (Figura 1).

As pacientes com linfonodos considerados negativos pela ultrassonografia foram selecionadas para a biópsia do linfonodo sentinela, mesmo que no exame clínico a axila estivesse positiva.

Aquelas com linfonodos suspeitos pela ultrassonografia foram submetidas à biópsia por agulha fina (PAAF - agulha de 22 gauge e seringa de $10 \mathrm{~mL}$ ), da região cortical alterada do linfonodo com maior dilatação da cortical. O grupo que apresentou PAAF negativas para metástase axilar foi encaminhado para a biópsia do linfonodo sentinela. As que apresentaram PAAF positiva para neoplasia foram encaminhadas para dissecção axilar.

A associação entre as variáveis foi calculada por meio dos testes do $\chi^{2}$ ou exato de Fisher. A seguir foram calculadas a sensibilidade, a especificidade, o valor preditivo positivo (VPP) e o valor preditivo negativo (VPN).

A análise de concordância entre o exame clínico, a ultrassonografia e a ultrassonografia associada com a biopsia por agulha fina foi realizada aplicando o cálculo do índice Kappa de Cohen.

Para as características da espessura da cortical, foi obtido o melhor valor por meio da curva Receiver Operator Characteristic (ROC).

Toda a análise estatística foi realizada com o emprego do programa Statistical Analysis System for Windows ${ }^{\circledR}$, versão 9.1, considerando-se nível de significância de 5\% ou o valor $\mathrm{p}$ correspondente.

\section{Resultados}

$\mathrm{Na}$ avaliação clínica das axilas, 128 foram consideradas clinicamente normais $(74,8 \%)$ e $25,2 \%$, clinicamente suspeitas. Com a ultrassonografia, 101 axilas foram consideradas suspeitas $(59, \%)$ e 70 , negativas $(41, \%)$. No estudo anatomopatológico dos linfonodos axilares, foi observado que $65(38,1 \%)$ axilas foram positivas ( 5 casos de micrometástases ) e $106(61,9, \%)$, negativas.

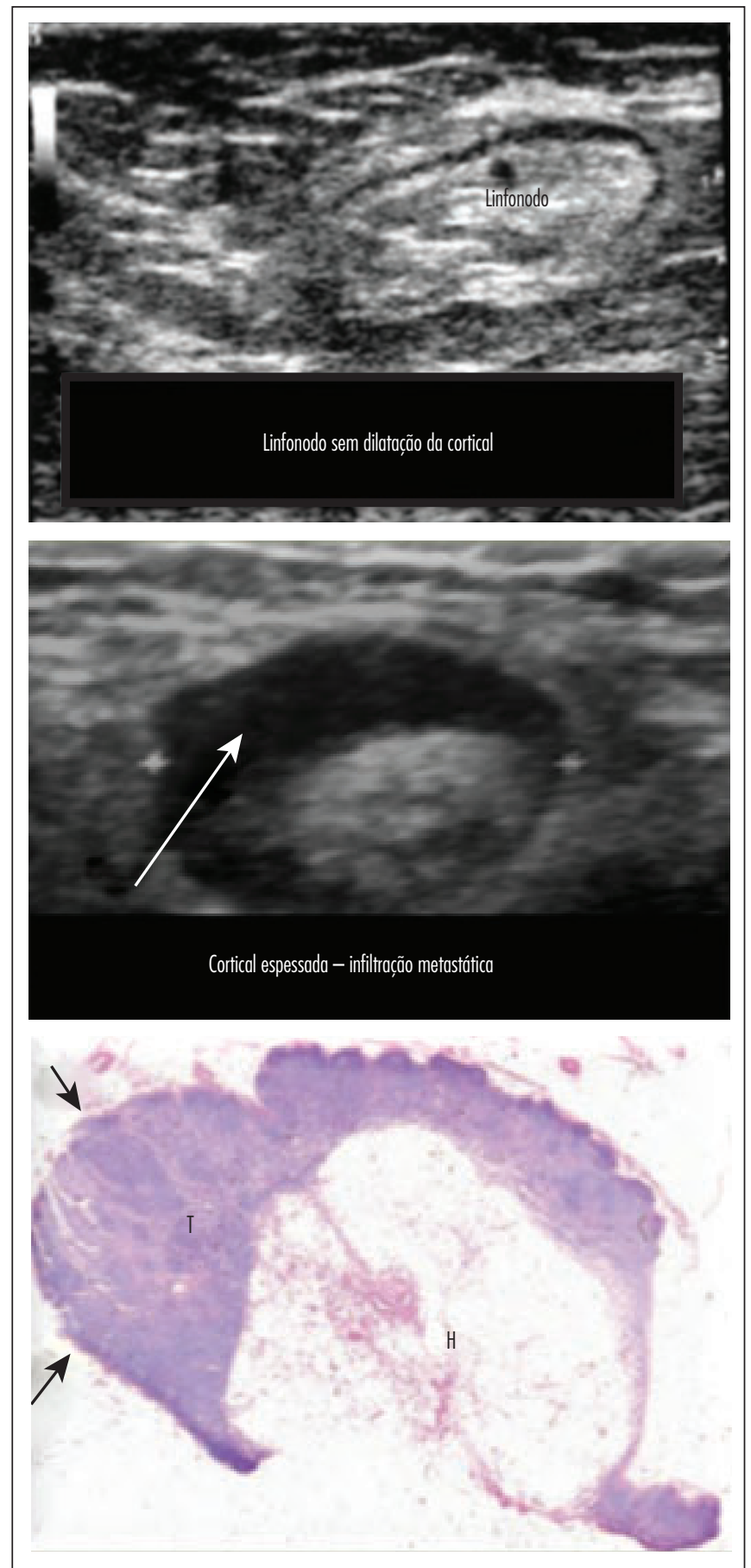

Figura 1. (A) Linfonodos considerados negativos pela ultrassonografia (sem dilatação da cortical), (B) Linfonodo considerado positivo (com espessamento da cortical maior que $2,3 \mathrm{~mm}$ ) e respectivo corte histológico.

Foram realizadas 121 BLS e em 15 o resultado foi positivo ( 5 casos de micrometástases). As pacientes submetidas a biopsias do linfonodo sentinela eram aquelas para as quais a ultrassonografia axilar foi negativa ou que a ultrassonografia foi positiva, mas a biópsia por agulha fina, negativa. Na Tabela 1 observamos a correlação anatomopatológica do linfodo axilar em relação à avaliação clínica e ultrassonográfica da axila. 
Tabela 1. Correlação entre a biopsia por agulha fina (em 101 axilas suspeitas pela ultrassonografia ${ }^{*}$ ) com os achados histopatológicos

\begin{tabular}{|c|c|c|c|c|c|c|c|}
\hline & \multicolumn{2}{|c|}{$\begin{array}{l}\text { Histologia } \\
\text { negativa }\end{array}$} & \multicolumn{2}{|c|}{$\begin{array}{l}\text { Histologia } \\
\text { positiva }\end{array}$} & \multicolumn{2}{|c|}{ Total } & \multirow{2}{*}{$\begin{array}{c}\text { Valor } \\
\mathbf{p}\end{array}$} \\
\hline & $n$ & $\%$ & n & $\%$ & $\mathrm{n}$ & $\%$ & \\
\hline PAAF Negativa & 41 & 100 & 10 & 16,7 & 51 & 50,5 & $<0,0001$ \\
\hline \multirow[t]{2}{*}{ PAAF Positiva } & 0 & 0 & 50 & 83,3 & 50 & 49,5 & \\
\hline & 41 & 100 & 60 & 100 & 101 & 100 & \\
\hline
\end{tabular}

PAAF: biopsia por agulha fina.

\#Das 171 axilas avaliadas pela ultrassonografia, 101 foram selecionadas para PAAF.

Tabela 2. Índice de Kappa para 0 exame clínico, a ultrassonografia e a ultrassonografia combinada a biopsia por agulha fina, com seus respectivos valores preditivos positivos, negativos e acurácia

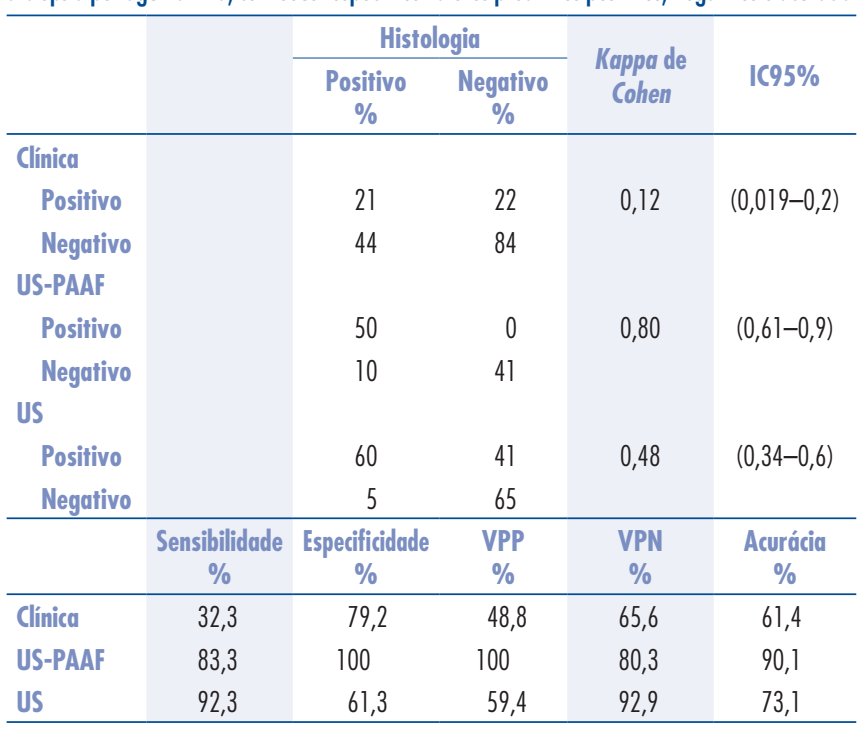

US: ultrassonografia; PAAF: biopsia por agulha fina; VPP: valor preditivo positivo; VPN: valor preditivo negativo.

Em relação ao exame clínico e à avaliação do estado axilar, observamos uma sensibilidade de $32,3 \%$, especificidade de $79,2 \%$, valor preditivo positivo de $48,8 \%$, valor preditivo negativo de $65,6 \%$ e acurácia de $61,4 \%$. Com a ultrassonografia axilar, a sensibilidade foi de $92,6 \%$, a especificidade, $61,3 \%$, o valor preditivo positivo, $59,4 \%$, o valor preditivo negativo, $92,8 \%$, e a acurácia, $73 \%$.

Os linfonodos com alteração na cortical foram submetidos à biopsia por agulha fina guiada pela ultrassonografia. Na Tabela 2 mostramos a relação entre o resultado da punção com o exame histopatológico dos linfonodos. A US-PAAF apresentou sensibilidade de $83,3 \%$, especificidade de $100 \%$, valor preditivo positivo de $100 \%$, preditivo negativo de $82 \%$ e acurácia de $90 \%$. O índice Kappa de Cohen foi de 0,12 para o exame clinico (considerado baixo), 0,48 para o US (considerado moderado) e 0,80 para US-PAAF( considerado ótimo).

Em relação à avaliação da região cortical do linfonodo pela ultrassonografia, realizamos uma curva ROC (Figura 2)

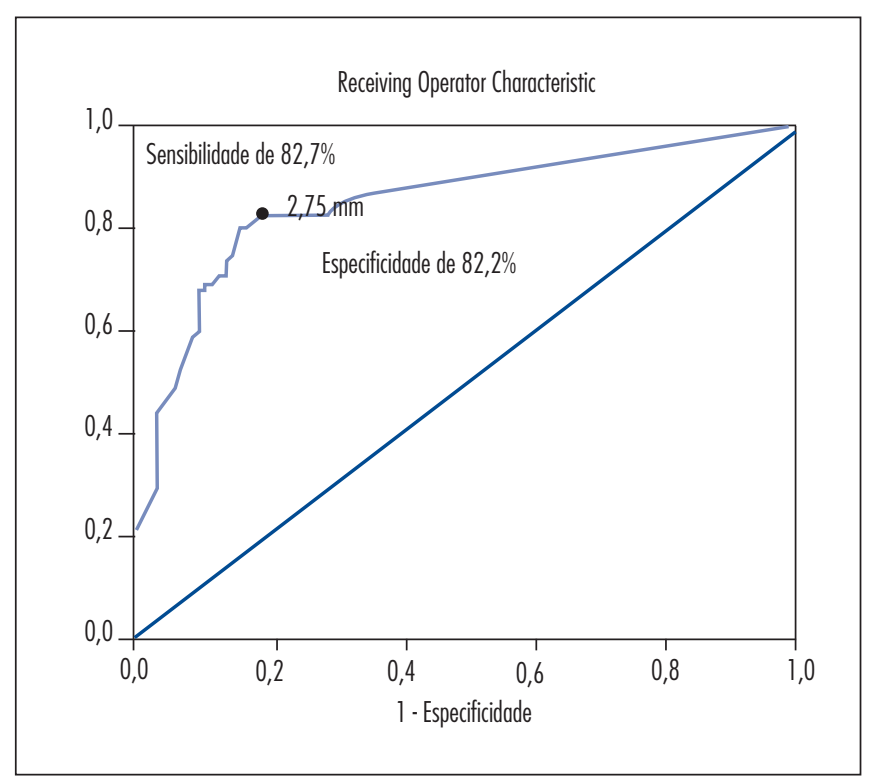

Figura 2. Curva Receiving Operator Characteristic avaliando o espessamento mostrando o valor com maior sensibilidade e especificidade.

para determinar um ponto de corte de maior sensibilidade e especificidade. Encontramos uma sensibilidade de $82,7 \%$ e especificidade de $82,2 \%$ para axila positiva no anatomopatológico quando a cortical tem valor de $2,75 \mathrm{~mm}$.

\section{Discussão}

A técnica do linfonodo sentinela representa um grande avanço para a avaliação axilar e o estadiamento; porém, a seleção das pacientes que irão ser submetidas a esse método continua rudimentar. As pacientes são classificadas, na maioria das vezes, em axilas negativas ou positivas apenas com o exame clínico. A falta de exatidão do exame clínico para avaliação axilar é conhecida: somente 63 a $66 \%$ das axilas classificadas como negativas são verdadeiramente negativas, e essa dificuldade aumenta para as pacientes obesas ${ }^{18}$.

Encontramos com o exame clínico um valor preditivo negativo de 65,6\%. Das 128 pacientes com axila clinicamente negativas que seriam selecionadas para o linfonodo sentinela, $44(35,4 \%)$ já teriam axila positiva, aumentando as chances de sentinelas falso negativos, além de submeter a paciente a um procedimento desnecessário e, nos casos da utilização do azul patente, ainda aumenta os riscos de reações alérgicas importantes. Também o índice Kappa de Cohen de apenas 0,18 é considerado pobre. Em concordância com esses dados, citamos o estudo de Milão, no qual 259 pacientes com tumores com menos de $2 \mathrm{~cm}$ de diâmetro e axilas clinicamente negativas foram selecionadas para a biópsia do linfonodo sentinela. Nesse grupo, 92 pacientes $(35,5 \%)$ tiveram o linfonodo positivo ${ }^{19}$. 
Um método simples e acessível para avaliação axilar e para reduzir o número de linfonodos sentinelas positivos é a utilização da ultrassonografia. Vários estudos demonstram os benefícios da avaliação axilar por essa técnica ${ }^{20-22}$.

Porém, devemos salientar que esse exame é operador e aparelhos dependentes. Dessa forma, melhores resultados são obtidos com observador experiente e aparelho de alta resolução. $\mathrm{Na}$ avaliação do linfonodo, valorizamos a alteração na região cortical, levando em consideração a fisiopatologia da metástase linfonodal. As células se alojam primeiro na cortical, dilatando-a e distendendo-a, assim, independente do tamanho ou da forma do linfonodo, é possível observar as alterações e irregularidades nessa região ${ }^{17,23}$.

É ressaltada nesta avaliação a utilização das imagens em harmônica digitalmente codificadas, que possibilita identificar a região cortical que fica nitidamente mais hipoecogênica, diferenciando-se da medular (hiperecogênica) e da gordura ao redor (isoecogênica). Isso contribuiu muito para a identificação e avaliação dos linfonodos.

O exame ultrassonográfico mostrou uma sensibilidade de $92,6 \%$ e uma especificidade de $61,0 \%$, com valor preditivo positivo de $59,8 \%$ e valor preditivo negativo de $92,8 \%$. É difícil comparar nossos dados com os da literatura, porque observamos uma grande variação na sensibilidade (54 a 95\%) e especificidade ( 44 a 91\%), mesmo quando limitamos as comparações a trabalhos prospectivos que utilizam critérios morfológicos e transdutores de alta frequência ${ }^{20,24,25}$.

Em todos esses trabalhos foi demonstrada a correlação entre a avaliação axilar pela ultrassonografia e o estado anatomopatológico da axila. Encontramos um alto valor preditivo negativo, ou seja, das 70 pacientes classificadas como axilas negativas pela US, apenas $5(7,2 \%)$ apresentaram axila positiva. Estes dados são também concordantes com esses estudos.

Quando associamos a avaliação ultrassonográfica com a biopsia por agulha fina, obtivemos uma redução no número de linfonodos sentinelas positivos em 63,6\%, ou seja, de 44 para 15 casos. Essa diferença de 29 casos reflete o diagnóstico ultrassonográfico antecipado do comprometimento axilar das pacientes que estavam com axila negativa clinicamente e que seriam submetidas à pesquisa do linfonodo sentinela. Se pensarmos nos altos custos com as técnicas de medicina nuclear e com a avaliação anatomopatológica do linfonodo sentinela intraoperatória, além das chances de falsos negativos desta avaliação e dos riscos de alergia com azul patente, temos uma ideia melhor dos benefícios dessa seleção ${ }^{26,27}$.Portanto, diminuímos os custos e selecionamos melhor as pacientes que serão submetidas à pesquisa do linfonodo sentinela.

Encontramos 101 axilas com linfonodos classificados como suspeitos pela ultrassonografia, e esses linfonodos foram submetidos à PAAF guiada pelo ultrassom, para pesquisarmos a presença de metástases. $\mathrm{O}$ valor preditivo positivo da US-PAAF foi de $100 \%$, mais alto que o da US $(68,3 \%)$. Portanto, a chance da axila positiva ser detectada é muito maior se associarmos a US com a PAAF. Isso fortalece a US como ferramenta de triagem do estado axilar, pois a chance de a axila ser positiva se o exame ultrassonográfico for negativo é pequena. No entanto, uma vez que o exame seja suspeito, é necessário o uso da PAAF, que tem valor preditivo positivo mais elevado que a US. Esses achados são semelhantes aos encontrados em outros estudos ${ }^{26,27}$.

Yang et al. ${ }^{28}$ foram os primeiros a indicarem uma espessura da cortical de 1 a $2 \mathrm{~mm}$ como valor normal. Posteriormente, o trabalho de Deurloo et al. ${ }^{25}$ definiu como espessura limite normal até $2,3 \mathrm{~mm}$, com especificidade de $44 \%$ e sensibilidade de $95 \%$. Neste optamos por empregar o critério de Deurloo et al. ${ }^{25}$. Assim, linfonodos com cortical homogênea com valor menor ou igual a 2,3 $\mathrm{mm}$ de espessura eram considerados normais e os linfonodos com cortical acima de 2,3 mm, suspeitos. Porém, para estabelecer um ponto de corte de maior sensibilidade e especificidade, construímos uma curva ROC da espessura da cortical determinando o valor de $2,75 \mathrm{~mm}$ com uma sensibilidade de $82,7 \%$ e especificidade de $82,2 \%$ para axila positiva no anatomopatológico. Se utilizarmos como valor de corte $2,3 \mathrm{~mm}$, a sensibilidade é maior $(92,6 \%)$, porém a especificidade é reduzida para $61,3 \%$. Assim, para examinadores que não estão familiarizados com esses critérios, sugerimos considerar o ponte de corte mais alto de $2,75 \mathrm{~mm}$, que é de mais fácil visualização e, para os examinadores mais experientes, considerar o de $2,3 \mathrm{~mm}$, que, apesar da especificidade menor, tem um alto valor preditivo negativo.

Existem outros parâmetros que podem ser utilizados para indicar anormalidade nos linfonodos, como diâmetro máximo, arredondamento anormal e alterações do fluxo pela doplervelocimetria, porém são anormalidades encontradas, na maioria das vezes, na doença metastática linfonodal avançada, na qual o exame clínico é eficiente devido aos linfonodos serem facilmente palpáveis ${ }^{29}$.

Concluímos que a utilização da ultrassonografia associada à biopsia por agulha fina para a avaliação do estado axilar e seleção das pacientes submetidas ao linfonodo sentinela traz real benefício para as pacientes, poupando-as de procedimentos desnecessários e permitindo programar melhor as suas terapêuticas, além de se tratar de um método relativamente simples e econômico.

A ultrassonografia em conjunto com a biopsia por agulha fina mostrou-se útil como ferramenta para o estudo axilar e, consequentemente, para a seleção das pacientes que serão submetidas à biópsia do linfonodo sentinela, evitando 63,7\% de biópsias desnecessárias do linfonodo sentinela. 
1. Morton DL, Wen DR, Wong JH, Economou JS, Cagle LA, Storm FK, et al. Technical details of intraoperative lymphatic mapping for early stage melanoma. Arch Surg. 1992;127(4):392-9.

2. van Rijk MC, Deurloo EE, Nieweg OE, Gilhuijs KG, Peterse JL, Rutgers EJ, et al. Ultrasonography and fine-needle aspiration cytology can spare breast cancer patients unnecessary sentinel lymph node biopsy. Ann Surg Oncol. 2006;13(1):32-5.

3. Krag D, Weaver D, Ashikaga T, Moffat F, Klimberg VS, Shriver $C$, et al. The sentinel lymph node in breast cancer:a multicenter validation study. N Engl J Med. 1998;339(14):941-6.

4. Doting MH, Jansen L, Nieweg OE, Piers DA, Tiebosch AT, Koops $\mathrm{HS}$, et al. Lymphatic mapping with intralesional tracer administration in breast carcinoma patients. Cancer. 2000;88(1 1):2546-52.

5. Schaapveld M, Otter R, de Vries EG, Fidler V, Grond JA, van der Graaf WT, et al. Variability in axillary lymph node dissection for breast cancer. J Surg Oncol. 2004;87(1):4-12.

6. Canavese G, Catturich A, Vecchio C, Tomei D, Gipponi M, Villa $G$, et al. Sentinel node biopsy compared with complete axillary dissection for staging early breast cancer with clinically negative lymph nodes:results of randomized trial. Ann Oncol. 2009;20(6):1001-7.

7. Krag DN, Anderson SJ, Julian TB, Brown AM, Harlow SP, Ashikaga $T$, et al. Technical outcomes of sentinel-lymph-node resection and conventional axillary-lymph-node dissection in patients with clinically negative breast cancer:results from the NSAPB B-32 randomised phase III trial. Lancet Oncol. 2007;8(10):881-8.

8. Bobin JY, Spirito C, Isaac S, Zinzindohove C, Joualee A, Khaled $M$, et al. Le marquage lymphatique et la biopsie du ganglion sentinelle axillaire dans 243 cancers invasifs du sein sans ganglion palpable. Expérience du centre hospitalier Lyon-Sud. Ann Chir. 2000;125(9):861-70.

9. Boolbol SK, Fey JV, Borgen PI, Heerdt AS, Montgomery LL, Paglia M, et al. Intradermal isotope injection:a highly accurate method of lymphatic mapping in breast carcinoma. Ann Surg Oncol. 2001;8(1):20-4.

10. Bruneton JN, Caramella E, Héry M, Aubanel D, Manzino JJ, Picard $J \mathrm{~J}$. Axillary lymph node metastases in breast cancer:preoperative detection with US. Radiology. 1986;158(2):325-6.

11. Tate JJ, Lewis V, Archer T, Guyer PG, Royle GT, Taylor I. Ultrasound detection of axillary lymph node metastases in breast cancer. Eur J Surg Oncol. 1989;15(2):139-41.

12. Vaidya JS, Vyas JJ, Thakur MH, Khandelwal KC, Mittra I. Role of ultrasonography to detect axillary node involvement in operable breast cancer. Eur J Surg Oncol. 1996;22(2): 140-3.

13. Abe H, Schmidt RA, Sennett CA, Shimauchi A, Newstead GM US-guided core needle biopsy of axillary lymph nodes in patients with breast cancer:why and how to do it. Radiographics. 2007;27 Suppl 1:S91-9.

14. Lam WW, Yang WT, Chan YL, Stewart IE, Metreweli C, King W. Detection of axillary lymph node metastases in breast carcinoma by technetium- $99 \mathrm{~m}$ sestamibi breast scintigraphy, ultrasound and conventional mammography. Eur J Nucl Med. 1996;23(5):498-503.

15. Yang WT, Ahuja A, Tang A, Suen M, King W, Metreweli C. High resolution sonographic detection of axillary lymph node metastases in breast cancer. J Ultrasound Med. 1996;15(3):241-6.
16. Verbanck J, Vandewiele I, De Winter H, Tytgat J, van Aelst F, Tanghe $W$. Value of axillary ultrasonography and sonographically guided puncture of axillary nodes: a prospective study in 144 consecutive patients. J Clin Ultrasound. 1997;25(2):53-6.

17. Bedi DG, Krishnamurthy R, Krishnamurthy S, Edeiken BS, Le-Petross $H$, Fornage $B D$, et al. Cortical morphologic features of axillary lymph nodes as a predictor of metastasis in breast cancer:in vitro sonographic study. AJR Am J Roentgenol. 2008;191(3):646-52.

18. Zgajnar J, Hocevar M, Podkrajsek M, Hertl K, FrkovicGrazio S, Vidmar G, et al. Patients with preoperatively ultrasonically uninvolved axillary lymph nodes:a distinct subgroup of early breast cancer patients. Breast Cancer Res Treat. 2006;97(3):293-9.

19. Veronesi U, Paganelli G, Viale G, Luini A, Zurrida S, Galimberti $\mathrm{V}$, et al. A randomized comparison of sentinel-node biopsy with routine axillary dissection in breast cancer. N Engl J Med. 2003;349(6):546-53

20. Sapino A, Cassoni P, Zanon E, Fraire F, Croce S, Coluccia C, et al. Ultrasonographically-guided fine-needle aspiration of axillary lymph nodes:role in breast cancer management. $\mathrm{Br} J$ Cancer. 2003;88(5):702-6.

21. Rao R, Lilley L, Andrews V, Radford L, Ulissey M. Axillary staging by percutaneous biopsy:sensitivity of fine-needle aspiration versus core needle biopsy. Ann Surg Oncol. 2009; 16(5): 1 170-5.

22. Tahir M, Osman KA, Shabbir J, Rogers C, Suarez R, Reynolds T, et al. Preoperative axillary staging in breast cancer saving time and resources. Breast J. 2008;14(4):369-71.

23. Damera A, Evans AJ, Cornford EJ, Wilson AR, Burrell HC, James $\mathrm{JJ}$, et al. Diagnosis of axillary nodal metastases by ultrasoundguided core biopsy in primary operable breast cancer. $\mathrm{Br} J$ Cancer. 2003;89(7):1310-3.

24. Stavros AT. Breast ultrasound. Philadelphia:Lippincott Williams \& Wilkins;2004.

25. Deurloo EE, Tanis PJ, Gilhuijs KG, Muller SH, Kröger R, Peterse JL, et al. Reduction in the number of sentinel lymph node procedures by preoperative ultrasonography of the axilla in breast cancer. Eur J Cancer. 2003;39(8):1068-73.

26. Bonnema J, van Geel AN, van Ooijen B, Mali SP, Tjiam SL, Henzen-Logmans SC, et al. Ultrasound-guided aspiration biopsy for detection of nonpalpable axillary node metastases in breast cancer patients:new diagnostic method. World J Surg. 1997;21 (3):270-4.

27. Popli MB, Sahoo M, Mehrotra N, Choudhury M, Kumar A, Pathania $\mathrm{OP}$, et al. Preoperative ultrasound-guided fine-needle aspiration cytology for axillary staging in breast carcinoma. Australas Radiol. 2006;50(2): 122-6.

28. Yang WT, Ahuja A, Tang A, Suen M, King W, Metreweli C. Ultrasonographic demonstration of normal axillary lymph nodes:a learning curve. J Ultrasound Med. 1995;14(1 1):823-7.

29. Yang WT, Chang J, Metreweli C. Patients with breast cancer:differences in color Doppler flow and gray-scale US features of benign and malignant axillary lymph nodes. Radiology. 2000;215(2): 568-73. 